\title{
Des intentions de l'auteur aux usages en classe, première réflexion sur la cohérence des usages d'une ressource
}

Between designing and use, how to address the question of resource's consistency?

\section{Gilles Aldon, Mathias Front et Marie-Line Gardes}

\section{OpenEdition \\ Journals}

Édition électronique

URL : https://journals.openedition.org/educationdidactique/2810

DOI : $10.4000 /$ educationdidactique.2810

ISSN : 2111-4838

\section{Éditeur}

Presses universitaires de Rennes

\section{Édition imprimée}

Date de publication : 31 décembre 2017

Pagination : $9-30$

ISBN : 978-2-7535-7319-2

ISSN : 1956-3485

\section{Référence électronique}

Gilles Aldon, Mathias Front et Marie-Line Gardes, «Des intentions de l'auteur aux usages en classe, première réflexion sur la cohérence des usages d'une ressource », Éducation et didactique [En ligne], 11-3 | 2017, mis en ligne le 31 décembre 2019, consulté le 17 août 2022. URL : http:// journals.openedition.org/educationdidactique/2810 ; DOI : https://doi.org/10.4000/ educationdidactique.2810 


\title{
DES INTENTIONS DE L'AUTEUR AUX USAGES EN CLASSE, PREMIÈRE RÉFLEXION SUR LA COHÉRENCE DES USAGES D'UNE RESSOURCE
}

\author{
Gilles Aldon \\ IFÉ-ENS de Lyon, EA S2HEP \\ Mathias Front \\ ESPE de l'académie de Lyon, EA S2HEP \\ Marie-Line Gardes \\ ESPE de l'académie de Lyon, UMR 5304, Institut des sciences cognitives, université de Lyon
}

Instrumenté par une ressource, un enseignant développe son propre projet pour la classe. En tant que concepteurs de ressources, nous avons souhaité présenter dans ce texte, un cadre pour l'analyse de la convergence entre les projets des auteurs de ressources et ceux des enseignants utilisant ces ressources. Nous utiliserons les cadres de la genèse documentaire et de la structuration des milieux d'une situation didactique pour concevoir un indicateur de cette convergence qui nous permettra de définir le concept de cohérence de l'usage d'une ressource ; une ingénierie didactique a été construite sur une méthode d'étude des écarts entre les intentions des auteurs de la ressource et les réalisations de l'enseignant. Nous rendons ensuite compte de la mise à l'épreuve de cette méthode dans le cadre d'une expérimentation qui questionne tous les niveaux de la situation didactique considérée. Lanalyse fait apparaître des éléments qui montrent l'utilisabilité de ce concept de cohérence et son usage possible dans des champs plus vastes.

Mots-clés : cohérence de l'usage d'une ressource, genèse documentaire, structuration des milieux, dimension expérimentale, situations de recherche en classe, situations didactiques.

\section{Between designing and use, how to address the question of resource's consistency?}

Instrumented by a resource, a teacher develops his/her own project for the class. As designers of resources, we wanted to present in this paper a framework for the analysis of the convergence between the authors' projects and those of teachers using these resources. We use the framework of the documentational genesis and structuring milieux in a didactical situation to define an indicator of convergence that will allow us to define the concept of resource's consistency; a didactical engineering has been built in order to study the differences between the intentions of the authors of a resource and the teacher's achievement in the classroom. Then we give an account of the test of this method in the context of an experiment that examines all levels of the teaching situation. The analysis reveals elements which show the usability of the concept of consistency and its possible use in larger fields.

Keywords: resource's consistency, documentational genesis, structuring milieux, experimental approach, research situations in the classroom, didactical situation. 


\section{INTRODUCTION}

La réflexion sur les ressources, leur création, leurs usages, leur qualité n'est pas nouvelle mais prend une importance de plus en plus grande lorsque la diversité de l'offre et la modification des supports des ressources imposent des choix. Les travaux du collectif Réseau Thématique Pluridisciplinaire sur le DOC (Pédauque, 2006) montrent les phénomènes de « redocumentarisation » portés par les développements du numérique, et la genèse documentaire (Gueudet \& Trouche, 2008, 2009, 2010) tente de modéliser les phénomènes didactiques liés à la documentation des professeurs de mathématiques. Différents projets ou recherches prennent en compte l'appropriation par les utilisateurs potentiels des ressources produites, en développant par exemple, des notions de qualité des ressources (Trgalova \& Leyraud, 2010 ; Bourgeat et al., 2010) qui interrogent à la fois la validité épistémologique des contenus et l'efficacité pédagogique des ressources. Les processus d'intégration d'une ressource dans le système documentaire d'un enseignant, modélisés par les concepts d'instrumentation et d'instrumentalisation, traduisent les liens existants entre les intentions, souvent implicites, portées par la ressource et celles du professeur. C'est précisément pour mettre au jour ces processus et préciser leur nature que nous avons suivi une ressource particulière, depuis sa conception jusqu'à son utilisation dans la construction d'une séquence de classe. Une telle étude nécessite à la fois la construction d'un cadre théorique et la mise en œuvre d'une ingénierie spécifique. Dans un premier temps, nous montrerons comment à partir des cadres proposés par la genèse documentaire et par la théorie des situations didactiques (Brousseau, 1998), nous pouvons définir le concept de cohérence de l'usage d'une ressource, puis nous décrirons l'ingénierie mise en place pour une première mise à l'épreuve de ce concept que nous analyserons à partir des observations menées dans ce cadre.

\section{DE LA COHÉRENCE DE L'USAGE D'UNE RESSOURCE}

\section{Introduction à la notion de cohérence}

Le concept de cohérence dans le curriculum (Newmann et al., 2001 ; Schmidt et al., 2005 ;
Boning, 2007) conduit à décrire dans quelle mesure les différents outils mis à disposition des enseignants (les manuels, les programmes, etc. dans une institution), s'accordent avec leurs pratiques :

"One term used to describe a particular type of coherence is alignment, by which is meant the degree to which various policy instruments available to the system, e.g. standards, textbooks, and assessments, accord with each other and with school practice." (Schmidt et al., 2005, p. 527.)

Ces recherches tentent de définir la cohérence globale d'un curriculum et mettent en évidence l'importance d'une cohérence entre les contenus des disciplines scolaires, la façon de présenter les concepts et les disciplines académiques.

"We suggest that standards are coherent if they specify topics, including the depth at which the topic is to be studied as well as the sequencing of the topics, both within each grade and across the grades, in a way that is consistent with the structure of the underlying discipline." (Ibid., p. 554.)

Par ailleurs, la cohérence interne dans les pratiques des enseignants fait également l'objet d'études comme en témoigne le récent article de Mangiante-Orsola (2012) qui présente la cohérence comme une construction propre à chaque individu et l'étudie dans la relation entre la tâche prescrite et la tâche effective dont témoigne l'activité de l'enseignant dans un contexte particulier. La cohérence apparaît alors comme une logique interne au cœur des pratiques des enseignants :

« Il s'agit donc d'examiner les systèmes de réponses que celui-ci produit face aux contraintes du métier pour en dégager une certaine cohérence, c'est-à-dire, une certaine logique au cœur même de ses pratiques. » (Op. cité, p. 295.)

La cohérence de l'activité d'un enseignant est ainsi vue comme " une harmonie, un rapport logique, une absence de contradiction dans l'enchaînement des parties d'un tout $»(\mathrm{TLFi})^{1}$.

Dans cet article, nous nous intéressons à l'enrichissement du système documentaire d'un enseignant par l'intégration d'une ressource et aux relations des intentions des auteurs de cette ressource aux usages qui en sont faits dans l'enseignement. Le concept de 
cohérence tel qu'il est exprimé dans les définitions précédentes s'intéresse à une logique globale dans un curriculum ou dans les pratiques des enseignants. Il ne permet pas en revanche de conceptualiser les rapports existant entre une ressource, nécessairement construite avec les intentions des auteurs, et ses usages.

Dans cette perspective nous proposons une définition de la cohérence de l'usage d'une ressource afin d'identifier des éléments fondamentaux pour leur analyse ou leur conception. Pour modéliser cette cohérence, nous nous appuierons sur l'articulation de deux cadres théoriques : la genèse documentaire (Gueudet \& Trouche, 2008) et la structuration des milieux dans la théorie des situations didactiques (Brousseau, 1986, 1998).

\section{Genèse documentaire}

La multiplication des ressources pour le travail du professeur, notamment avec le développement de l'internet, a conduit à prolonger l'approche instrumentale en considérant les ressources disponibles comme des artefacts. L'approche documentaire (Gueudet \& Trouche, 2008, 2009, 2010) modélise les interactions des sujets avec les ressources à leur disposition. Le professeur intègre des ressources dans tout le processus d'élaboration de son enseignement, et construit ses propres documents reliés à une classe de situations, dans ce que Gueudet et Trouche (2010, p. 58) nomment le travail documentaire. À l'instar de la genèse instrumentale qui décrit la transformation d'un artefact en instrument comme le résultat d'un double mouvement, du sujet vers l'artefact et de l'artefact vers le sujet, le processus de transformation des « ressources » en « document », la genèse documentaire, est le résultat d'une instrumentalisation, sujets vers les ressources et d'une instrumentation, ressources vers les sujets.

L'instrumentalisation apparaît comme le façonnage, la mise en forme d'un ensemble de ressources pour les propres usages du sujet, l'instrumentation modifiant, elle, les comportements du sujet. À un instant donné, le document est alors la combinaison des ressources considérées et d'un schème d'utilisation dans un contexte donné. Le processus ne se termine pas, puisque les documents ainsi constitués peuvent être considérés comme de nouvelles ressources. Les ressources utilisées par les ensei- gnants sont des éléments de structuration de leurs actions dans la classe (Gueudet $\&$ Trouche, 2008), ce qui fait écho à la notion de cohérence de la pratique des enseignants étudiée par MangianteOrsola (2012). Le processus de genèse documentaire relie une instrumentalisation de ces ressources aux conceptions des enseignants vis-à-vis à la fois des mathématiques, de l'apprentissage et de l'enseignement. En ce sens, et c'est ce qui nous intéresse ici, la genèse documentaire donne des outils pour suivre l'évolution d'une ressource dans le milieu de l'enseignant dans une situation de construction de séance de classe. Tout comme Rabardel (1999) définit des systèmes d'instruments comme «l'ensemble organisé des moyens disponibles pour l'activité du sujet en fonction des tâches et des contextes $»$ (p. 212), il est également important de noter qu'un document ne vit pas d'une façon isolée mais fait partie d'un ensemble de documents qui s'articulent les uns aux autres dans un système documentaire (Gueudet \& Trouche, 2009). Dans ce contexte, les processus d'instrumentation et d'instrumentalisation traduisent les échanges entre les intentions de la ressource et celles du professeur à l'intérieur d'un système documentaire donné. Mais pour mettre au jour ces processus il est alors nécessaire de mettre à l'épreuve de la contingence le document développé à partir de la ressource et d'observer la mise en œuvre de la situation proposée par l'enseignant. Nous faisons l'hypothèse que c'est à travers l'observation de cette mise en œuvre que nous pourrons analyser l'interprétation des intentions de la ressource par le professeur et notamment, à travers le document qu'il aura construit pour appuyer la situation didactique. Nous faisons alors appel au cadre de la structuration des milieux pour comprendre et suivre, dans son travail documentaire, un professeur construisant et mettant en œuvre une situation didactique. La genèse documentaire, en se focalisant sur l'évolution des ressources, et la structuration des milieux, examinant la situation didactique du point de vue du professeur et des élèves, offrent des cadres complémentaires et féconds pour analyser les liens qui unissent ressource et usages.

\section{Structuration des milieux}

Dans le modèle de la structuration des milieux, proposé par Brousseau (1986) et prolongé par Margolinas (2004) et Bloch et Gibel (2011), le 
professeur est un acteur essentiel de la situation, aussi bien dans sa position de préparation, lorsqu'il est confronté au milieu didactique et suivants (cf. Tableau 1), que dans sa position d'observateur ou de professeur. Dans ce modèle, la situation didactique (SO) est toutefois centrale et c'est la raison pour laquelle notre méthodologie s'appuiera sur la mise en œuvre effective d'une situation dans un contexte particulier, après étude de l'activité de préparation du professeur.

L'étude d'une situation didactique conduit ainsi à considérer l'environnement tout entier du sujet apprenant, le professeur étant un des éléments de cet environnement. Le point de vue adopté et la dynamique de la situation modifient la perception du milieu et justifient la description structurée des milieux comme indiquée sur le Tableau 1. Dans notre observation, c'est le point de vue du professeur que nous considérerons et nous utiliserons la structure des milieux dans une analyse " descendante ", c'est-à-dire une analyse prenant le point de vue de l'enseignant depuis sa position dans la situation noosphérienne, confronté au milieu de construction dans une situation non finalisée. Il construit, dans cette posture, une articulation entre les savoirs, les savoirs à enseigner et ses conceptions épistémologiques relatives à sa discipline, à son enseignement et son apprentissage. Les ressources utilisées par le professeur constituent un élément du milieu de projet et évoluent progressivement dans les interactions entre le P-constructeur et le milieu de projet. Dans la situation de projet, le P-projeteur, en interaction avec le milieu didactique, construit le milieu matériel de la situation objective qu'il proposera aux élèves de la situation objective. Les ressources mobilisées se concrétisent en une ressource que la confrontation aux milieux sous-didactiques complétera en un document, en y intégrant, dans l'action, des modes d'utilisation qui se transformeront, dans l'usage, en schèmes faisant partie du document.

Des analyses croisées montantes et descendantes de situations didactiques montrent qu'il est possible et même fréquent qu'un décalage entre les intentions du professeur et les attentes des élèves se produise. Margolinas (2004) modélise cela par le concept de bifurcation didactique : lorsqu'il propose une situation aux élèves, le professeur projette son intention d'enseignement, c'est-à-dire sa volonté de modifier le système de connaissances des élèves. Il construit alors une situation didactique s'appuyant sur un milieu. Dans cette position, les élèves ignorent les intentions du professeur, ils peuvent cependant les imaginer (dans leur position d'E-réflexif, E+1) et projeter à leur tour leur propre situation adidactique en s'appuyant sur le milieu matériel de la situation objective. Il y a bifurcation lorsque, confrontés à ce milieu matériel, les élèves investissent une situation de référence différente de celle prévue dans les intentions du professeur. Plusieurs scénarios peuvent alors se dérouler :

- les élèves investissent une situation de référence reposant sur une situation nildidactique ; dans ce cas, ils font fonctionner des connaissances naturalisées sans en construire ou en rencontrer de nouvelles;

- les élèves investissent une situation de référence qui repose sur une situation objective

Tableau 1.

Tableau de la structuration des milieux, extrait de (Bloch, 2005, p. 55)

\begin{tabular}{|c|c|c|c|c|}
\hline M3 : Milieu de construction & & P3 : P-noosphérien & $\begin{array}{l}\text { S3: situation } \\
\text { noosphérienne }\end{array}$ & Sur didactique \\
\hline M2 : Milieu de projet & & $\mathrm{P} 2$ : P-constructeur & $\begin{array}{l}\text { S2 : situation de } \\
\text { construction }\end{array}$ & \\
\hline Ml : Milieu didactique & El : E-réflexif & P1 : P-projeteur & S1 : situation de projet & \\
\hline $\begin{array}{l}\text { M0 : Milieu d'apprentissage : } \\
\text { institutionnalisation }\end{array}$ & E0 : Élève & $\begin{array}{l}\text { P0 : Professeur } \\
\text { enseignant }\end{array}$ & So : situation didactique & \\
\hline $\begin{array}{l}\text { M-1 : Milieu de référence : } \\
\text { formulation validation }\end{array}$ & E-1 : E-apprenant & P-1 : P-régulateur & $\begin{array}{l}\text { S-1 : situation } \\
\text { d'apprentissage }\end{array}$ & a-didactique \\
\hline M-2 : Milieu objectif : action & E-2 : E-agissant & $\begin{array}{l}\text { P-2 : P-observateur, } \\
\text { dévolueur }\end{array}$ & $\begin{array}{l}\text { S-2 : situation de } \\
\text { référence }\end{array}$ & \\
\hline M-3 : Milieu matériel & E-3 : E-objectif & & S-3 : situation objective & \\
\hline
\end{tabular}


adidactique distincte de la situation prévue par le professeur, ou plus précisément extérieure aux intentions didactiques du professeur, permettant la naturalisation de nouvelles connaissances, mais en décalage avec l'institutionnalisation.

Le modèle de structuration des milieux permet de penser de façon différente les deux types de branches marginales: dans le cas d'une branche marginale adidactique, l'élève s'attend à voir institutionnaliser un savoir qui n'est pas dans le projet du professeur, dans le cas (plus fréquent) d'une branche marginale nildidactique, l'élève n'apprend rien de nouveau et le professeur comme l'élève se trouvent " décalés " dans une phase d'institutionnalisation des savoirs. Le professeur parce qu'il fait reposer son institutionnalisation sur des connaissances qui n'ont pas été mobilisées et l'élève parce qu'il reçoit un savoir sans relations avec les connaissances mises en œuvre dans la situation.

En lien avec les relations des acteurs et du milieu aux différents étages de la structure présentée, la notion d'incident didactique est définie comme un élément déterminant de la dynamique de la classe tant du point de vue du professeur que de celui des élèves (Aldon, 2011) : « un incident didactique est fondamentalement lié aux acteurs de la situation » (p. 26). En particulier, un incident « de contrat» est la rupture dans une situation donnée du contrat didactique, fondement de la situation, pouvant conduire à une bifurcation didactique, c'est-à-dire à l'investissement par les acteurs d'une situation adidactique différente de celle présente dans les intentions initiales de l'enseignant ou de l'Auteur.

Le modèle de structuration des milieux n'a pas été construit pour prendre en compte les auteurs de ressources. Pourtant, dans le processus de constructions de ressources, les auteurs sont partie prenante de l'ensemble des situations didactiques potentielles qui peuvent être construites en s'appuyant sur cette ressource. Nous proposons donc d'ajouter au modèle de la structuration des milieux un point de vue supplémentaire, celui de l'Auteur en interaction avec la structure des milieux de situations didactiques (cf. Fig. 1). C'est en partant de cet ajout au modèle que nous définissons dans le paragraphe suivant la cohérence de l'usage d'une ressource.
Figure 1.

Ajout du point de vue de l'auteur

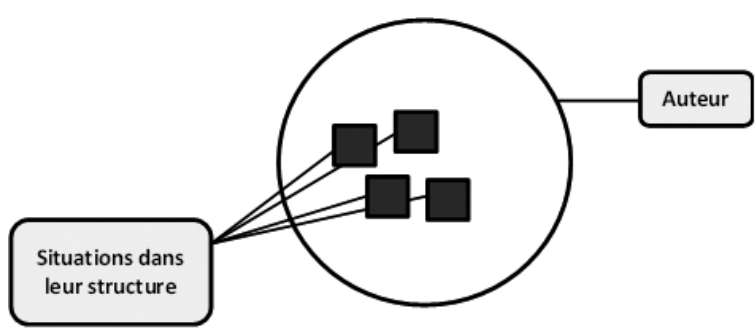

Cohérence de l'usage d'une ressource

Dans le modèle représenté Figure 1, l'Auteur correspond à l'ensemble des auteurs d'une ressource, avec leurs compétences propres. Les interactions entre Auteur et Professeur peuvent être réelles (c'est par exemple le cas des groupes de ressources IREM ${ }^{2}$, ou des professeurs associés à l'IFÉ ${ }^{3}$, ou des professeurs auteurs de manuel) ou imaginées.

Le milieu de l'Auteur, dans la situation de création de la ressource, est constitué de l'ensemble des situations qu'il imagine comme potentiellement construites à partir de la ressource ou issues d'expérimentations (cf. Fig. 2). Ainsi dans ce que nous appellerons « la ressource » est « embarqué » un ensemble de situations potentielles. Dans la construction et le suivi d'une ressource, l'Auteur est en interaction avec ce milieu.

Figure 2.

Milieu de l'Auteur dans la situation de création de la ressource

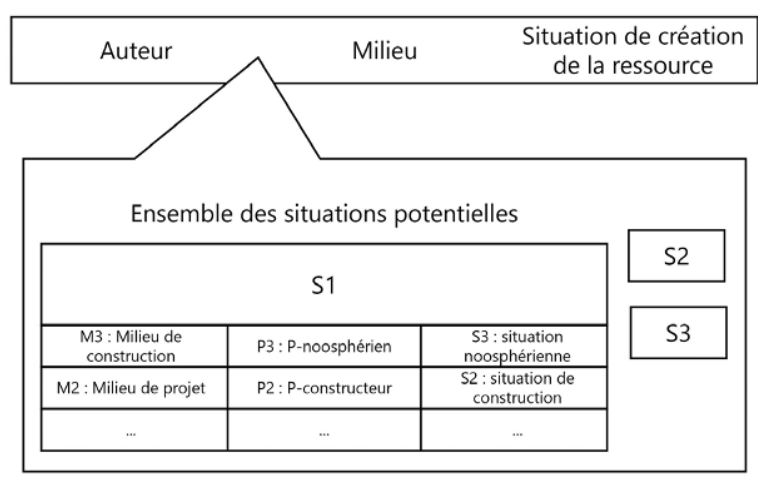

Une fois la ressource construite et publiée, c'est l'Auteur qui est absent des interactions entre le professeur et la ressource. Pour autant, à travers 
les interactions avec le milieu constitué des situations didactiques potentiellement portées dans la ressource, l'Auteur a construit, intégré et imprégné la ressource de ses intentions. Le professeur dans la situation de construction se confronte à la ressource et l'instrumentalise pour en faire un document d'appui d'une situation didactique. De ce fait, il interprète la ressource, comme un musicien interprète une œuvre, en traduisant les intentions de la ressource pour son projet.

Dans une situation noosphérienne, le P-noosphérien interagit avec le milieu de construction dans lequel ses ressources sont présentes. En particulier, le professeur interprète les intentions de l'Auteur d'une ressource à travers ses interactions avec la ressource. Dans les situations de construction et de projet, et en fonction de ses interprétations, le professeur construit en s'appuyant sur des éléments de la ressource une situation didactique. Suivant un processus similaire à celui des bifurcations didactiques explicitées dans le paragraphe précédent, la situation de construction peut porter en elle des intentions distinctes de celles de la ressource pour déboucher sur une situation didactique différente des situations didactiques envisagées par l'auteur de la ressource. Dans ce cas on parlera de bifurcation de construction. La cohérence de l'usage d'une ressource se juge alors par la non bifurcation de construction.

Ainsi il ne s'agit pas ici de cohérence épistémologique qui mettrait en relation un savoir visé et une situation ad-hoc. Dans la théorie des situations didactiques, le concept de situation fondamentale peut être compris comme un type de cohérence entre une situation et les savoirs visés ; dans la théorie anthropologique du didactique, les praxéologies peuvent aussi apparaître comme une mise en cohérence des types de tâches et des savoirs associés. Ici, pour nous qui confrontons les intentions des auteurs d'une ressource et les situations didactiques mises en œuvre en appui sur cette ressource, la cohérence sera jugée a posteriori. Ainsi la définition précédente ne peut s'entendre que comme qualifiant l'usage de la ressource dans la mise en œuvre de situations didactiques. Il s'agit donc d'une cohérence didactique, en lien avec la contingence. Et ceci permettra de s'assurer ou non de la cohérence des usages d'une ressource dans des contextes variés, sous des conceptions d'enseignement diverses.

Le cadre de la structuration des milieux nous permettra ainsi de juger de la cohérence de l'usage d'une ressource. La genèse documentaire donne quant à elle des outils pour suivre une ressource dans un processus d'intégration et de transformation dans l'ensemble des ressources utilisées par un enseignant dans son travail de préparation, d'orchestration et d'analyse d'une situation didactique. L'étude de la pertinence du cadre proposé nécessite ensuite de soumettre cette définition de la cohérence à l'épreuve d'une expérimentation ; c'est la raison pour laquelle nous présentons ci-dessous une ingénierie spécifique.

\section{INGÉNIERIE DIDACTIQUE POUR LA MISE À L'ÉPREUVE DE LA DÉFINITION DE LA COHÉRENCE DE L'USAGE D'UNE RESSOURCE}

Nous considérons ici une ingénierie didactique comme une méthodologie de recherche fondée sur une réalisation didactique, validée par une confrontation d'une analyse a priori et d'une analyse a posteriori. L'analyse a priori est construite autour de l'analyse par inspection de la ressource (Tricot et al., 2003), et d'un recueil des intentions de l'Auteur. Précisons donc ici que nous sommes amenés à considérer des ressources, conçues à des fins d'enseignement, pour lesquelles il est possible d'avoir accès aux intentions des auteurs, de façon plus ou moins explicite. L'analyse a posteriori est construite sur l'observation et l'analyse de la situation mise en œuvre. La confrontation entre ces deux analyses permet alors de conclure à la bifurcation de construction ou non, c'est-à-dire à la non cohérence ou à la cohérence de l'usage de la ressource.

Plus précisément, l'ingénierie est construite autour d'une ressource identifiée, la ressource EXPRIME (Aldon et al., 2010). L'ingénierie didactique (Artigue, 1988) que nous proposons, étudie trois aspects : l'affirmation des intentions des auteurs, la construction d'une situation par le professeur et enfin la mise en œuvre de cette situation dans une classe de Terminale Scientifique 4 .

Concernant le premier aspect, au-delà de l'analyse de la ressource numérique existante, dont nous donnons quelques éléments en annexe 1, pour mettre en évidence les intentions de l'Auteur de la ressource, nous rendons compte d'un court texte, rédigé par cet Auteur à l'occasion de notre recherche, dans lequel il développe des éléments du contexte et les hypothèses qui ont présidé à la création de la ressource. Pour les deux autres aspects, correspon- 
dant au suivi de l'usage de la ressource par un enseignant et à la mise en ouvre d'une situation dans la classe, nous avons proposé la ressource à un enseignant, ci-après désigné par JFC, avec la commande de la réalisation d'une séance de classe à sa convenance. Afin de recueillir des éléments sur la construction de séance à partir de la ressource, un entretien préalable a été réalisé. Cet entretien a eu lieu avant la séance de mise en œuvre en classe et a été enregistré. Pour l'observation de la séance tous les moyens nécessaires à la double observation de la pratique de l'enseignant et de l'activité des élèves lors de toutes les phases ont été utilisés: micros, micros cravate, dictaphones, caméras sur pied ou portées, prise de notes. Un entretien postérieur, permettant un retour d'usage, a également été réalisé et enregistré.

\section{Intentions de l'Auteur de la ressource EXPRIME}

Nous reprenons du texte de la ressource EXPRIME, les intentions affichées de l'Auteur. La ressource EXPRIME est une ressource sur cédérom qui propose un ensemble de textes théoriques, de propositions de mises en œuvre, de productions d'élèves, d'analyses... autour de situations de recherche de problèmes. L'auteur inscrit ses travaux dans une thématique explorée depuis de nombreuses années par l'IREM de Lyon, celle des problèmes de recherche pour la classe, notamment les problèmes ouverts (Arsac \& Mante, 1988, 2007).

Dans ce cadre, il est apparu nécessaire à l'Auteur de retravailler la relation aux objets mathématiques en affirmant leur position centrale pour la construction de savoirs. Il précise que pour Arsac et Mante (2008, p. 2), la naissance des " problèmes ouverts » se fonde sur une "idée [qui] ne se réfère qu'à la pratique des mathématiciens. Elle ne se fonde ni sur une théorie pédagogique, ni sur une théorie de l'apprentissage ». L'Auteur note que de la pratique qui résulte de cette idée, Arsac et Mante dégagent des effets « thérapeutiques» :

«Un changement du rapport des élèves aux mathématiques, un changement du rapport des élèves à l'enseignant de mathématiques, un changement du rapport des élèves entre eux. " (Arsac \& Mante, 2008, p. 7.)

L'Auteur admet avec Arsac et Mante l'existence de ces modifications de « rapport » et reprend les carac- téristiques de mises en œuvre de ces «problèmes ouverts » permettant ces changements qu'il retient comme favorables (Arsac \& Mante, 2007).

L'Auteur reprend le positionnement socioconstructiviste de ces précurseurs en précisant que, par socioconstructivisme, il entend une théorie socle nécessaire pour développer toute réflexion sur les modes de construction de connaissances chez l'élève. Affirmer que tout élève construit ses connaissances l'a ainsi conduit à produire des situations de recherche de problèmes où le milieu objectif est tel que l'élève agissant peut s'engager dans un processus amenant potentiellement à des constructions de connaissances, c'est-à-dire à l'intégration structurée d'éléments nouveaux dans un système de pensée en mouvement. Ceci implique en particulier, pour la situation, des phases de recherche individuelles. Pour l'Auteur, le rôle majeur et nécessaire des interactions sociales dans les élaborations de connaissances, demande également que les situations proposées intègrent des phases d'échanges, d'oppositions, de débats, qui forgent et enrichissent les élaborations mathématiques. L'auteur s'appuie alors sur le scénario du " problème ouvert » qui contient des phases de recherche individuelle puis en groupe, de débats intra-groupe, des temps de présentation de résultats collectifs, de débats inter-groupes et enfin un temps d'institutionnalisation. Pour l'Auteur, ce scénario a fait ses preuves dans l'évolution du rapport des élèves aux mathématiques, aux autres élèves, à l'enseignant. Mais il se demande s'il permet, ce pour quoi il n'a pas été fait : la construction de savoirs.

Pour l'Auteur, comprendre les potentialités du scénario de ce point de vue nécessite de questionner les mathématiques entendues dans la locution « rapport aux mathématiques ». Il interroge donc, dans un premier temps, l'activité de recherche en classe, le rapport aux objets mathématiques que cela induit et les élaborations mathématiques potentielles. Il se réfère à Polya (1945), Lakatos (1984) et Schoenfeld (1985) pour tenter d'identifier des invariants de l'activité de recherche d'un individu. Pour ce qui concerne l'activité de recherche en classe, il cite Arsac \& Mante (2007) et Perrin (2007) qui mettent en avant une démarche, désignée chez Perrin par «méthode expérimentale » et décrite ainsi :

«Elle comprend plusieurs étapes à répéter éventuellement : expérience, observation de l'expérience, formulation de conjectures, tentative de preuve, contre-expérience, production éventuelle de 
contre-exemples, formulation de nouvelles conjectures, nouvelle tentative de preuve, etc. » (Perrin, 2007, p. 5.)

Pour l'Auteur, cette approche modélisante a permis certaines analyses et propositions de dispositifs didactiques, mais il s'interroge pour savoir dans quelle mesure elle peut s'abstraire du regard indispensable sur les objets des expériences ? Selon lui, on ne peut se passer de questionner la relation aux objets en jeu si l'on veut s'assurer de la nature mathématique des produits d'une activité de recherche. L'Auteur indique que cette place des objets est particulièrement centrale du point de vue d'une dimension expérimentale, définie par Durand-Guerrier par

\footnotetext{
« le va-et-vient entre un travail avec les objets que l'on essaye de définir et de délimiter et l'élaboration et/ou la mise à l'épreuve d'une théorie, le plus souvent locale, visant à rendre compte des propriétés de ces objets » (Durand-Guerrier, 2006, p. 17.)
}

L'Auteur considère d'une part, que l'activité mathématique s'appuie sur un domaine d'expérience, constitué d'un ensemble d'objets suffisamment familiers pour permettre la prospection et, d'autre part, que la pertinence des objets produits dans l'activité se juge à un moment ou un autre, à la qualité des rétroactions qu'ils produisent sur le « réel ». Ainsi, pour lui, les essais, les formulations de conjectures, les tentatives de preuves, ne peuvent apparaître porteurs de sens que lorsque l'élève agissant sur des objets mathématiques ou certaines de ses représentations, matérielles ou non, produit de nouvelles connaissances, pertinentes au sens où elles permettent une nouvelle compréhension de la situation mathématique. Ainsi, pour l'Auteur, l'étude du rapport aux mathématiques ne peut se passer de la considération des actions sur les objets, du produit de ces actions et de l'analyse de la pertinence réelle et ressentie de l'activité, en termes d'efficacité dans la compréhension des phénomènes étudiés.

Cette centration sur l'activité mathématique fortement liée à des objets amène l'Auteur à mettre en avant des situations qui doivent favoriser les allers retours entre des expériences sur les objets mathématiques, des formulations de conjectures, des élaborations de raisonnements, de démonstration, d'algorithmes.

L'Auteur considère que les situations didactiques proposées doivent s'envisager plus comme des environnements propices au questionnement mathématique que comme des chemins menant inéluctablement soit au résultat attendu, soit éventuellement au mur dont seul l'enseignant maîtrise le dépassement. Il précise qu'elles doivent permettre une activité mathématique riche et particulièrement dans sa dimension expérimentale, qu'elles sont à la fois résistantes, au carrefour de champs de savoirs divers, et de dévolution aisée. Il ajoute que ces situations permettent de se confronter aux objets mathématiques et de tisser autour d'un objet à explorer une toile de relations et de significations.

Pour l'Auteur, faire vivre une situation qui permette d'atteindre de tels objectifs est déjà un succès, qu'il est nécessaire de mettre en évidence dès que la pratique dans la classe l'aura permis. Il considère, en effet, qu'il est fondamental de montrer aux élèves non seulement qu'ils ont su mettre en œuvre une pratique de recherche mais bien évidemment qu'elle est productive, et qu'il est possible d'insérer leurs propres constructions ponctuelles dans une compréhension plus globale des mathématiques. L'Auteur s'interroge alors : «Peut-on et est-il nécessaire d'aller plus loin en termes d'élaboration de savoirs ? ». Il indique que le débat est loin d'être tranché et cite Arsac et Mante:

« Il peut être contradictoire de vouloir viser à la fois le changement du rapport aux mathématiques et l'émergence d'une connaissance nouvelle. » (Arsac \& Mante, 2008, p. 7.)

Pour l'Auteur, il semble opportun d'être un peu moins tranché et de ne pas fermer la porte à des émergences, non seulement en termes de consolidation de savoirs en construction mais également de savoirs nouveaux. L'Auteur précise que des expériences ont en effet montré que les élaborations théoriques réalisées lors de situations de recherche, sont de l'ordre de la construction de savoirs nouveaux (Front, 2012).

\section{Analyse des milieux surdidactiques de la situation}

Dans ce paragraphe, il s'agit de confronter les intentions exprimées par l'Auteur avec la construction effective d'une séance de classe par JFC, le professeur qui a accepté de mettre en œuvre une séance de recherche de problème en s'appuyant sur la ressource EXPRIME. JFC est un enseignant expérimenté, proche 
de la retraite, développant une attitude réflexive sur son enseignement. Il n'a pas de proximité immédiate avec les travaux de l'Auteur de la ressource et, comme nous le verrons, il n'a pas de pratique en classe proche de celle envisagée par l'Auteur de la ressource. Le recueil des éléments permettant d'analyser comment JFC a construit sa séance d'enseignement, à partir de la ressource, s'appuie sur les entretiens, et plus particulièrement sur l'entretien préalable, mais aussi sur l'observation de classe et l'activité du professeur dans la situation didactique. Même si la position du professeur dans les différents niveaux n'est pas, par nature, temporelle, nous présenterons dans la suite les interactions du professeur avec la ressource successivement dans les situations $\mathrm{S}+3, \mathrm{~S}+2, \mathrm{~S}+1$, $\mathrm{S} 0$ et $\mathrm{S}-1$, étant bien entendu que l'activité à un moment donné du professeur relève de différents niveaux. Précisons également, en suivant (Margolinas, 2002, p. 12), que " dans les situations S0, S+1 et S+2, caractéristiques de l'activité du professeur, les milieux inf. sont des milieux antagonistes et les milieux sup. sont le plus souvent des milieux alliés».

\section{Usage de la ressource dans la situation noosphérienne}

En tant que P+3, JFC a accepté d'utiliser la ressource dans la situation noosphérienne c'est-àdire d'inclure dans son système documentaire propre la ressource EXPRIME. Les conceptions de JFC ont ainsi évolué en incluant de nouveaux schèmes développés dans l'usage de cette ressource, comme on le verra dans la suite en nous appuyant sur son discours mais aussi sur les observations dans la classe. Il n'est cependant pas possible dans cette observation de modéliser l'évolution des conceptions de JFC en termes d'invariants opératoires qui, par nature, se construisent dans le temps. C'est pourquoi l'analyse de l'usage de la ressource est construite sur une analyse descendante plutôt que sur les invariants opératoires dans les schèmes d'usage de cette ressource. En revanche $\mathrm{P}+3$, pour construire une situation pour sa classe, a parcouru toutes les situations de la ressource. Il a particulièrement consulté les savoirs mathématiques en jeu dans chaque situation comme il avait l'habitude de le faire dans son système de ressource propre.

«JFC : je les ai quand même analysés les uns après les autres mais je pense quand même que sur chacun d'eux il y a matière à faire des investigations. » (entretien préalable.)

« JFC : oui j'ai regardé sur chacun des problèmes j'ai regardé les savoirs mathématiques oui. » (entretien préalable.)

$\mathrm{P}+3$ veut choisir une situation permettant à ses élèves de Terminale de construire ou de conforter des savoirs mathématiques qui leur seront utiles pour le baccalauréat. Ainsi le choix de la situation de la ressource s'est spécifiquement appuyé sur les savoirs mathématiques en jeu dans les problèmes. JFC a ainsi exclu certaines situations sur ce critère :

«JFC : je m'étais dit ça, un exemple, telle situation, je ne peux pas l'exploiter avec mes élèves parce qu'il faut, les TS, parce qu'il faut l'arithmétique et j'en ai qu'un qui fait la spé math [...]. Donc j'ai cherché quelque chose qui ne, qui ne, enfin, pour lesquelles les connaissances étaient des connaissances que mes élèves avaient, par exemple, j'ai exclu aussi, mis à part l'arithmétique, un sujet qui pouvait conduire à faire l'usage des lois de probabilités continues, qu'on n'a pas encore fait, donc déjà, j'ai éliminé les sujets comme ça. » (entretien préalable.)

Le choix de la situation a ainsi nécessité, pour JFC, une première appropriation de la ressource qui s'est effectuée par la création d'un fichier dans lequel chaque situation est résumée en fonction des savoirs mathématiques en jeu et des savoirs mathématiques accessibles à ses élèves. Les processus d'instrumentation et d'instrumentalisation sont ici clairement en action.

Dans l'entretien, JFC revient sur l'apport de la rubrique de la ressource concernant les savoirs mathématiques liés aux problèmes étudiés. Il souligne que cela peut faire "gagner» du temps à l'enseignant en lui apportant un regard plus ouvert et plus riche sur le problème. $\mathrm{P}+3$ garde une distance et une vigilance vis à vis de l'instrumentation.

« JFC : c'est pas indispensable mais c'est vrai que c'est pas plus mal de de les avoir consultés, en tout cas pour le prof qui veut utiliser un de ces problèmes euh ça lui gagne du temps dans une certaine mesure et puis ça lui ouvre des horizons qu'il n'aurait peut-être pas découverts tout seul mais d'un autre côté [...] si on les regarde un peu prématurément en même temps on perd la spontanéité ou les idées qu'on pourrait avoir soi- même quoi. » (entretien préalable.) 
Il précise qu'anticiper sur la résolution mathématique du problème de recherche n'est pas indispensable pour le faire vivre en classe mais que de disposer de différents éléments à pouvoir communiquer est appréciable :

«JFC : avant de proposer un problème ouvert euh il me semble que [...] c'est pas une obligation que d'avoir anticiper [...], mais quand même quand on a une situation à proposer c'est quand même bien si, je crois, on a on a des éléments pour pouvoir [...] apporter un maximum de choses c'est vrai que si on n'a pas d'idée sur des suffisamment ouvertes et suffisamment riches on sera on sera peut-être moins intéressant. » (entretien préalable.)

À travers ces différentes citations, nous pouvons voir que les intentions des auteurs, quant à l'importance des savoirs mathématiques en jeu dans les problèmes de recherche, rencontrent les préoccupations de cet enseignant dans la situation noosphérienne.

Ainsi, dans le choix de la situation de recherche, le lien entre les savoirs mathématiques potentiels et ceux du programme est important. Apparaît également ici la richesse attendue des développements mathématiques possibles en situation au-delà de simples apports sur une notion bien délimitée.

Les réponses de JFC lors de l'entretien préalable, montrent en revanche que certaines rubriques n'ont pas influencé son choix de la situation. Par exemple, la rubrique des situations d'apprentissage ou des références :

« DK : les situations d'apprentissage, ça c'est les exemples des expérimentations qu'on a déjà faites dans nos classes ou dans des classes d'autres collègues.

JFC : Oui oui, je les ai moins regardées, ça m'intéressait un petit peu moins. »

Ainsi dans la situation noosphérienne, $\mathrm{P}+3$ conserve une liberté vis-à-vis des conceptions d'enseignement / apprentissage présentent dans le milieu.

\section{Usage de la ressource dans la situation de construction}

JFC a choisi la situation de "La rivière ». Mathématiquement, il s'agit d'un problème d'optimisation : minimiser une somme de distances dans le plan. La ressource propose une analyse mathématique du problème en détaillant trois méthodes de résolution. La première méthode est géométrique et utilise la symétrie axiale, la seconde est une interprétation physique du problème et utilise la réflexion et la troisième méthode est analytique et consiste à étudier la fonction qui exprime la distance à minimiser. La ressource contient également une analyse a priori didactique, avec notamment l'analyse des variables de la situation. Enfin, elle présente différents comptes rendus d'expérimentation en classe (collège, lycée et université) qui montrent les procédures diverses et variées mises en œuvre par les élèves et les étudiants ${ }^{5}$.

JFC utilise la ressource pour construire sa propre situation. $\mathrm{P}+2$ souhaite laisser le maximum d'ouverture au problème pour permettre aux élèves d'investir des connaissances diverses et en tout cas non directement liées au cours de la classe. Concernant la formulation de l'énoncé du problème, il précise dans l'entretien préalable qu'il ne pense pas ajouter d'information supplémentaire par rapport à l'énoncé présenté dans la ressource :

« JFC : [...] de laisser les, le problème très ouvert donc je ne vais sûrement pas renforcer les informations, ce n'est pas du tout dans mon intention. »

L'énoncé qui sera présenté aux élèves sera effectivement repris d'une formulation de la ressource, issue d'une expérimentation en classe de Première Scientifique ${ }^{6}$ (dans la rubrique Situations d'apprentissage).

Figure 3.

Énoncé du problème de la rivière proposé aux élèves

Un cavalier s'apprête à rentrer à l'écurie. Toutefois, il doit encore faire boire son cheval à la rivière proche (à cet endroit la rivière coule quasiment de façon rectiligne). Par ailleurs, il souhaite économiser sa monture. À quel endroit de la rivière doit-il faire boire son cheval pour avoir la plus petite distance possible lors du trajet de retour à l'écurie?

À cette formulation, JFC a ajouté un dessin, « une sorte de mathématisation du problème " (JFC lors de la séance de classe) représentant la rivière, le cavalier et l'écurie, adapté d'un schéma de la ressource : 
Figure 4.

Dessin de JFC illustrant l'énoncé du problème de la rivière proposé aux élèves

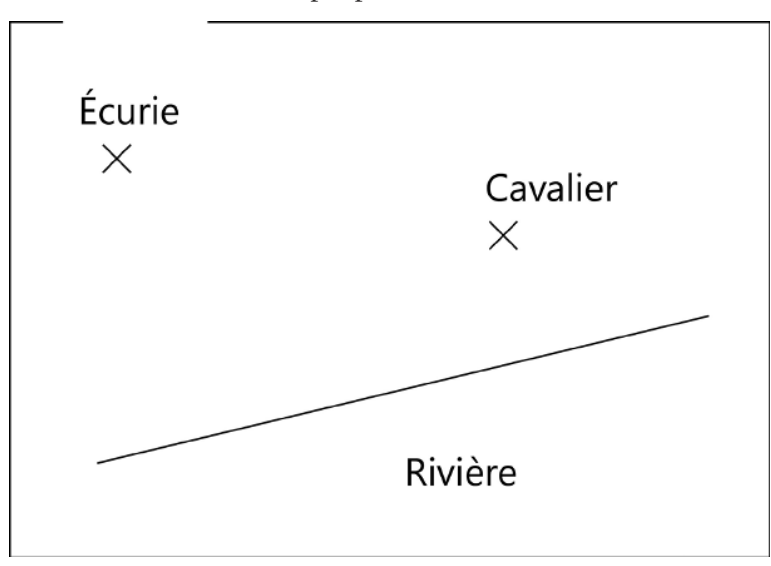

Cette adaptation de l'énoncé initial est le témoin d'une instrumentalisation de la ressource par JFC. $\mathrm{P}+2$ modifie la proposition de la ressource, tout en conservant les aspects essentiels de la formulation du problème de recherche.

Pour construire la situation de la rivière pour sa classe, JFC dit avoir utilisé la ressource « entre 50 et $75 \% »$. Dans l'entretien postérieur, il explicite précisément ce qu'il a fait à partir de la ressource mais aussi comment cette ressource s'incluait dans son système de ressource :

« JFC : J'ai utilisé la ressource comment dire euh à quel pourcentage j'ai utilisé la ressource on va dire à entre 50 et 75 pourcent quand même c'est-à-dire que j'ai repris pour la solution analytique j'ai essayé de faire les calculs moi aussi [...] j'ai pris ma calculatrice calcul formel et puis j'ai quand même regardé à la main j'ai vu que ça se faisait aussi à la main pour une part hein y a une équation du second degré à résoudre j'ai vu que s'arrangeait bien qu'ils peuvent le faire heu voilà bon je me suis refait ma figure GeoGebra. »

Ces extraits montrent que $\mathrm{P}+2$ a construit sa situation par des allers et retours entre la ressource et ses propres réflexions sur le problème conduites dans son système de ressources; ici la calculatrice formelle et le logiciel Geogebra qui s'articulent avec l'usage de la ressource. Son projet de construction de situation le mène plutôt à faire les calculs à la main et à établir un lien avec les connaissances de ses élèves. Ce dernier point semble constituer un critère important pour la construction de sa situation. Il se place dans le rôle de ses élèves pour évaluer les difficultés potentielles dans la résolution du problème.
La construction de sa séance s'est également appuyée sur sa consultation des situations connexes de la ressource. Dans l'entretien préalable, JFC pense les utiliser pour préparer des prolongements à la situation au cas où certains élèves avanceraient rapidement dans la résolution du problème.

«JFC : je pense que je vais utiliser la ressource pour préparer les prolongements au cas où certains aillent vite. »

Après avoir consulté les situations connexes autour de la situation de la rivière, JFC se rend compte qu'elles ne sont pas à la portée de ses élèves mais semble avoir été intéressé par les informations données dans cette rubrique.

«JFC : donc la ressource je l'ai trouvé très utile et j'ai regardé les situations connexes d'ailleurs je me suis dit huu ils n'iront pas jusque-là mais c'est vrai que bon ça peut être intéressant et j'ai bien apprécié l'interprétation physique. » (entretien postérieur.)

Dans l'entretien postérieur, il dit penser à présenter cette interprétation physique (trajet d'un rayon de lumière) à ces élèves, une occasion de faire le lien avec leur cours d'optique. Du point de vue du professeur, et même s'il n'utilise pas ces prolongements dans la situation, la confrontation à la ressource amène $\mathrm{P}+2$ à modifier son système de connaissances qui pourra être réutilisé dans une nouvelle situation de construction. La rubrique « situations connexes » joue donc ici un rôle d'un élément d'un milieu antagoniste ; $\mathrm{P}+2$ reçoit de la ressource une rétroaction non prévue mais qui le conduit à adapter et à modifier son projet. Cette partie de la ressource a ainsi été une aide à la construction de la séance par JFC, notamment pour circonscrire la situation pour ses élèves. Mais elle a également été une source de prolongements potentiels de la situation pour l'enseignant. Elle semble ainsi remplir les objectifs initiaux des auteurs de la ressource.

\section{Usage de la ressource dans la situation de projet}

Dans la situation de projet, $\mathrm{P}+1$ a alors utilisé la ressource plus spécifiquement pour préparer la mise en œuvre de la situation S0 dans la classe. Dans un premier temps, il a recours à la ressource pour construire le diaporama qui sera présenté aux élèves en début de séance. 
«JFC : Donc je vais préparer les documents ce weekend et du coup, je vais à nouveau prendre la ressource d'une part pour préparer le document que je vais leur remettre pour amorcer la recherche. » (entretien préalable.)

Ce diaporama reprend en très grande partie les éléments de la ressource mais $\mathrm{P}+1$, en relation avec sa position noosphérienne, va, sur un point spécifique, proposer à ses élèves de s'engager dans une réflexion métacognitive avec laquelle il les familiarise depuis deux ans. $\mathrm{P}+1$ dans cette situation interagit ainsi avec le milieu SO = M1 mais aussi avec les milieux surdidactiques supérieurs.

Dans un second temps, JFC consulte particulièrement la rubrique «Gestion d'une séance » de la ressource pour préparer la mise en œuvre dans sa classe :

«JFC : oui ben je pense que déjà elle constitue un moyen euh éducatif pour le prof puisque le prof par exemple qui regarde la $\mathrm{BD}^{7}$ euh il apprend de manière en même temps humoristique euh ça lui donne des idées si il en a s'il en a pas trop et en tout cas ça lui permet d'affiner ce qu'il pouvait avoir en tête pour euh pour animer sa séance et puis y a des réflexions qui sont aussi parfois présentées comme un petit peu amusantes et qui sont pas inintéressantes. » (entretien préalable.)

La façon dont JFC se projette dans la mise en œuvre de la séance est relativement fidèle au contenu de cette rubrique, comme en témoigne l'extrait suivant :

« JFC : donc une présentation rapide, à mon avis moins de 10 minutes, hein, puisque j'ai vu que souvent c'était 10 minutes pour expliquer comment ça doit se passer, euh probablement d'ailleurs sur le document que je vais leur donner je leur ai mis le timing en leur disant qu'ils ont 50 minutes ou une heure pour faire les recherches, en rappelant que dans la dernière partie de leur recherche ils doivent rédiger un petit compte-rendu sur un transparent que je leur fournirai. » (entretien préalable.)

On peut apercevoir, là encore ce double mouvement d'instrumentalisation et instrumentation dans la préparation de sa séance de classe : instrumentalisation puisqu'il va construire un document, reprenant a priori l'esprit de la ressource mais en l'agrémentant d'éléments supplémentaires qui lui sont propres et instrumentation puisque la ressource le conduit à faire des choix spécifiques, choix de la salle informatique par exemple. Concernant son rôle lors de la mise en œeuvre de la situation dans la classe, JFC détermine, également les conditions de ses éventuelles interventions et leurs contenus lors des phases de recherche collective puis lors de la phase de débat :

« JFC : j'essaierai d'être très prudent avec ce que je dis, c'est-à-dire, de ne pas donner de, d'informations mathématiques mais simplement, éventuellement des indications pour les aider à se remettre ou à exploiter ce qu'ils ont déjà trouvé, à faire des remarques sur ce qu'ils ont déjà ou mettre en valeur ou en évidence ce qu'ils ont éventuellement trouvé si je vois qu'ils sont bloqués sinon si ça, si ça roule tout seul tant mieux et puis donc, au terme d'une heure dix puisqu'il y a la présentation plus une heure etc, euh, je pense que je leur demanderai de se regrouper pour qu'on puisse faire une présentation de travail des groupes. » (entretien préalable.)

Ainsi sa lecture de la ressource semble traduire une interprétation conforme aux intentions des auteurs.

\section{Retour sur la contingence à partir de l'observation de la mise en ouvre dans la classe}

Nous revenons ici sur le produit du projet du professeur instrumenté par la ressource : une séance en classe portée par le projet décrit précédemment. Pour la suite, nous choisissons de décrire brièvement le travail de deux groupes dans les phases adidactiques et de pointer quatre actions de l'enseignant. Ceci doit permettre de rendre compte des caractéristiques du produit de l'enseignant suivant plusieurs niveaux d'analyse.

\section{Activité et production de deux groupes d'élèves}

Cette description de l'activité des deux groupes entre nécessairement dans le processus de confrontation entre l'analyse a priori et la mise en œuvre de la situation. La ressource EXPRIME et son Auteur, construisent leur proposition d'enseignement des mathématiques autour de la recherche de problèmes, en développant l'autonomie des élèves à travers des activités à dimension expérimentale, dans des phases adidactiques. Il est donc indispensable pour juger de la cohérence de l'usage de la ressource dans la situation construite et jouée par le professeur d'observer l'activité des élèves, sans prendre parti sur le bienfondé des intentions de l'Auteur. 


\section{Activité et production du groupe 1}

Le groupe 1 est composé de trois élèves dont deux (E1 et E2) s'engagent très franchement dans la recherche et un (E3) reste plus en retrait. Le temps de recherche individuelle de 10 minutes environ permet à E1 et E2 de produire chacun des représentations différentes de la situation. El comprend mal la contrainte de minimisation de la somme des distances mais en s'appuyant sur un modèle simplifié à l'excès produit des calculs de distance. E2, lui, construit un modèle correct et produit des figures modélisant la situation. Le temps collectif qui suit permet une confrontation des points de vue qui s'enrichissent mutuellement. La question du but à poursuivre sera tranchée en faveur de E2, mais on observe rapidement, alors que El est prêt à déchirer sa production, E2 s'appropriant les résultats partiels de E1. La suite du travail du groupe se développe dans deux directions. E2 et E3 construisent une figure dynamique sous GeoGebra et émettent la conjecture de l'existence d'une unique solution au problème. L'outil logiciel leur permet de conforter la validité de la question retenue, d'observer la variabilité des distances et de la somme. El s'engage dans du calcul algébrique en appui sur ses premiers écrits. Le travail du groupe au complet se prolonge jusqu'à la fin de la séance sur la production d'expressions algébriques puis fonctionnelles, la dernière expression produite permettant d'envisager, par un calcul de variations, une voie vers une solution à la question posée (cf. l'affiche du groupe en annexe 2).

On observe en particulier pour l'activité de ce groupe une appropriation rapide des enjeux de la situation de recherche avec un engagement dans des manipulations d'objets familiers et des avancées dans l'étude du problème. Les confrontations à l'intérieur du groupe et la mise en œuvre rapide de l'outil algébrique, suite à une incompréhension initiale de l'énoncé, sont clairement des éléments facilitateurs.

\section{Activité et production du groupe 2}

Le groupe 2 s'engage lui sur de très nombreuses pistes géométriques puis métriques. Une exploration de la situation leur permet tout d'abord d'ouvrir une piste barycentrique :

« On a essayé de... en bougeant les points A et B de voir ce que ça faisait. Dès qu'on change un point, ça change. Plus la distance $\mathrm{b}$ est petite, plus le point d'intersection sur la rivière se rapproche du point, son projeté, ça nous a rappelé la notion de barycentre, un petit peu, le point de la rivière, serait le barycentre des points $\mathrm{C}$ et $\mathrm{E}$, on n'est pas arrivé à développer. »

Le groupe a également produit des conjectures permettant de localiser le point solution :

« et après, on a essayé une méthode plus géométrique, en essayant de faire des cercles, des tangentes, on voyait que le point c'était, il était toujours à peu près compris entre les tangentes au cercle...»

mais a également produit des résultats utilisant la géométrie du triangle :

« on a essayé aussi de le faire avec des distances... j'ai essayé avec des vecteurs aussi, des normes... on a essayé pas mal de choses...»

Ces divers aspects de leur recherche sont visibles sur l'affiche du groupe (cf. annexe 3).

\section{Mise en ouvre par JFC : le lancement de l'activité}

Lors de l'entretien postérieur, JFC revient sur plusieurs éléments, la rubrique «Gestion d'une séance », en particulier la BD, la mise en œuvre de sa situation et un oubli dans sa présentation de la situation aux élèves :

«JFC: par contre dans la ressource il y a une question, je le disais tout à l'heure aussi, j'ai oublié, j'avais une diapo qui était "questions" et j’ai oublié de passer ma diapo et ça c'était mentionné dans la ressource, j'avais bien noté que je devais le faire et j'aurai dû le faire parce que l'énoncé a pas été bien compris par tous (entretien postérieur)

[...]

oui voilà, y avait une diapo il n'y avait qu'un titre avezvous des questions, donc là ils m'auraient probablement posé la question sur la compréhension de l'énoncé et donc bon là j'ai peut-être mal utilisé les informations dans la ressource. »

JFC fait référence à un conseil de gestion de classe présent dans la ressource qui préconise de faire lire l'énoncé et de demander aux élèves s'ils ont des questions sur l'énoncé du problème, l'Auteur donnant comme objectif à cette phase de s'assurer que les termes de l'énoncé sont compris par tous afin que chacun puisse s'engager dans l'activité attendue. JFC se souvient de cette remarque et d'avoir mentionné 
son importance. C'est en appui sur la ressource qu'il revient sur sa gestion de séance et pointe cet oubli.

\section{Mise en ouvre par JFC : le dessin proposé dans l'énoncé}

JFC, lors de l'entretien postérieur, se rend compte également que la présentation de son dessin n'aide pas forcément les élèves à introduire une paramétrisation et pourrait a contrario s'élever en obstacle, du fait du non parallélisme de la rivière avec le bord inférieur de la diapositive et des représentations des élèves sur les repères orthonormés du plan (cf. Fig. 4) :

«JFC : c'est pour ça que le fait simplement d'avoir mis un dessin comme j'en ai mis un j'ai mis un bout de dessin avec une droite et deux points euh peut-être que j'ai fait un pas qui les a peut-être pas aidés peutêtre certains oui peut-être d'autres non euh voilà peutêtre fallait-il leur dire aussi qu'ils avaient des initiatives à prendre sur euh la conceptualisation de la figure. » (entretien postérieur.)

\section{Une intervention marquante de JFC pendant la recherche collective}

Après avoir lancé la séance, surveillé le travail individuel, déclenché, observé et accompagné le travail des groupes, cinquante-six minutes après le début de la séance, JFC regarde sa montre, interrompt la recherche de la manière suivante :

«JFC : bien hein, excusez-moi, je vais vous interrompre un petit instant, vous m'accordez votre attention, s'il-vous-plait, tout le monde, s'il-vousplait... donc je pourrais vous laisser continuer, comme ça, certains ont fait des avancées, vous êtes en train de travailler sur vos conjectures, mais il vous reste encore un petit peu de temps, si on veut... bien employer ce temps, je crois que je dois vous donner quelques indications, je vois que vous arrivez nombreux à buter sur une petite difficulté c'est que, la figure que je vous ai proposée, j'aurais pu la faire différemment... les longueurs, vous l'avez compris déjà, plusieurs d'entre vous, n'ont aucune importance, ce qui est important, c'est de trouver un mode de construction de ce fameux point, et ce qui vous manque à tous, donc je vous fais remarquer, je vous donne cette information, c'est de choisir les paramètres ou des données pour définir votre figure, je vais aller un peu plus loin, pour vous aider...»
JFC explicite alors pendant 3 minutes et 40 secondes le besoin qu'il perçoit dans les travaux des élèves de l'introduction d'un paramétrage et plus globalement d'une identification des invariants. JFC s'appuie sur le schéma initial pour développer ses indications :

« vous pouvez choisir des paramètres, qui sont par exemple, la distance du point $\mathrm{A}$, si on l'appelle $\mathrm{A}$, à la droite, un premier paramètre... d'accord, un second paramètre pour l'autre point, ça ne suffit pas encore à définir la configuration, il faut encore définir la distance entre les deux projections orthogonales donc, je vous donne cette information, c'est une façon de procéder, qui va dans le sens de nos recherches je vois que vous butez un peu là-dessus donc fixez-vous trois lettres... a, $\mathrm{b}$ et $\mathrm{c}$, et puis je pense que ça vous aidera à avancer un petit peu, donc je vais le noter ici...»

Dans la production de quatre des groupes, on ne notera aucune référence explicite à ce point de vue et pour le groupe 1 , il est difficile de savoir dans quelle mesure cette intervention a fait évoluer la production qui était déjà bien avancée sur un aspect analytique avant l'interruption.

\section{Phase de présentation des transparents de chaque groupe}

JFC, une heure et neuf minutes après le début de la séance, débute une phase collective pendant laquelle un élève de chaque groupe vient présenter ce qui a été rédigé sur un transparent. JFC s'est positionné un mètre environ derrière le rétroprojecteur. Pendant le discours de chaque élève, il encourage pour que la parole soit fluide, valide quelques propositions, demande parfois une approbation de la salle. Cette phase, qui dure 12 minutes environ, est une phase de mise en commun mais dans les faits, sans débat sur la validité des propositions des élèves contrairement à ce qu'envisagent les auteurs de la ressource en référence à (Arsac \& Mante, 2007, p. 28). Elle permet de diffuser à tous les groupes les démarches engagées par chacun d'entre eux, donc de mettre au jour les conjectures, mais aussi les résultats partiels et les nouvelles connaissances. L'observation ne permet pas de savoir dans quelle mesure ces informations sont reçues par chaque élève, ni si une nouvelle culture commune est ainsi créée, permettant le prolongement de la réflexion sur le problème pour chacun. 


\section{RETOUR SUR LA COHÉRENCE}

Dans la situation donnée, nous étudions la cohérence de l'usage de la ressource en appui sur une analyse descendante et sur l'analyse d'un incident didactique. Précisons que, concernant le concept d'incident didactique, nous nous appuierons sur les travaux de Aldon (2011), repris dans (Aldon, 2014) qui se distinguent des travaux d'ergonomie cognitive (Rogalski, 2000 ; Roditi, 2001 ; Owen, 2007) ou de didactique (Clark-Wilson, 2010) par l'ancrage dans le milieu d'une situation.

\section{Première analyse}

L'étude de la cohérence de l'usage résulte de la confrontation des intentions de l'Auteur de la ressource et de la mise en ouvre de la situation didactique construite à partir des propositions de la ressource. Il n'est donc ici pas question d'analyser la pertinence de ces propositions mais plutôt de chercher dans cette confrontation les possibles bifurcations.

Dans la situation noosphérienne, JFC considère que les problèmes jouent un rôle dans l'apprentissage des élèves même si la façon dont il propose les problèmes diffère, dans son esprit, de ce que devraient être des "problèmes ouverts ", au sens des auteurs de la ressource. Mais les contraintes qu'il subit ou qu'il se donne dans l'institution l'empêchent de mettre en place une organisation pédagogique particulière :

«J'ai fait des choses, euh, quelques problèmes qui étaient un petit peu ouverts, on va dire, par contre, avec une organisation de classe qui n'est pas un travail en groupe, mais une organisation classique...» (entretien préalable.)

JFC affirme clairement sa difficulté à envisager la progression du curriculum prescrit sous cette pratique d'enseignement :

« je pense que ce genre d'attitude ça vient avec l'expérience sur ce genre de problème euh voilà donc le refaire je pense que ce serait pas mal si on avait le temps mais j'essaierai de le refaire peut-être une fois mais je crois que je pourrai pas le faire davantage pour des questions de timing. » (entretien postérieur.)
Il fait également l'hypothèse que l'élève doit revenir lui-même sur ses apprentissages pour évoluer, pour en tirer un profit général, ce que JFC développe longuement dans l'entretien après la séance et qu'il a intégré à sa présentation du problème (cf. Fig. 5).

Figure 5.

Extrait du diaporama de présentation du problème par JFC

\section{Et pour finir}

Cet exercice de recherche n'est-il pas une bonne occasion de mettre en œuvre la gestion mentale, éventuellement des phases de langage intérieur, bref n'est-il pas une bonne occasion de mettre votre comportement métacognitif au service de votre recherche, à la fois dans les recherches de stratégies de réponse et dans la gestion de votre comportement de recherche?

J'espère que vous me raconterez par la suite votre aventure cognitive.

Comme ceci a été évoqué précédemment, JFC cherche à développer un point de vue métacognitif chez ces élèves. Ce sont ses connaissances propres qui émergent ici, telles qu'elles sont apparues dans la mise en ouvre. Cette position épistémologique forte, enrichit le milieu idéologique, $\mathrm{M}+2$ composante sup (Margolinas, 2002, p. 15). JFC, comme projeteur, est donc épistémologiquement situé, ce qu'il confirme en affirmant :

«Oui mais sur la démarche ce que tu dis c'est vrai simplement c'est parce que ils sont, j'allais dire formatés, ces élèves mais j'exagère un peu mais je veux dire ils ont pas été assez ouverts. » (entretien postérieur.)

Pour JFC, la ressource est un élément du milieu de projet que le P-constructeur peut utiliser pour construire la situation. Il analyse dans la situation de construction les problèmes proposés de façon à rendre compatible ses conceptions de l'enseignement et le problème qu'il veut choisir :

«Euh, j’ai essayé de retenir quelque chose, un sujet, pour lequel ils soient moins déroutés et qu'ils soient davantage à même de faire des choses quoi. » (entretien préalable.)

Il ne perd pas de vue cependant que le problème doit être utile pour son enseignement et que cette pratique peut s'intégrer et servir son enseignement : 
« Ouais bon j'ai l'impression qu'ils ont bien travaillé ils ont été très coopératifs ils ont bien travaillé euh j’ai l'impression aussi que si je leur faisais faire ça trois quatre fois par exemple j'aurais pas les mêmes soucis que pour ce problème là où ils ont pas eu le réflexe de paramétrer je pense que ce genre d'attitude ça vient avec l'expérience sur ce genre de problème. » (entretien postérieur.)

Dans cet extrait d'interview après la séance, JFC revient sur ce qu'il considère comme un manque dans l'analyse préalable de la situation : le paramétrage du problème qu'il aurait souhaité voir appliquer plus spontanément et que l'on retrouve dans la situation S-1 (voire S-2) d'apprentissage lorsqu'il tente de proposer un paramétrage du problème à partir des propositions des élèves. En résumé, la ressource, comme élément du milieu de construction permet à P-constructeur d'envisager, pour ses élèves, une situation favorable à la prise d'initiatives et susceptible de leur faire travailler des éléments importants (considérés comme tels) du programme de terminale.

Dans la situation de projet, $\mathrm{P}+1$ utilise la ressource pour prévoir l'organisation didactique de la séance, en appui sur le problème choisi et sur les séances déjà proposées :

« je leur ai montré un problème ouvert celui des deux droites qui se coupent en dehors de la feuille et je leur ai dit ben voilà un exemple de problème ouvert du coup ça les a branchés, ils ont cherché un peu on en a reparlé etc., je leur ai proposé la solution et ça tombait bien parce qu'on venait de traiter l'homothétie avec les complexes donc en plus ça tombait pile poil voilà » (entretien postérieur.)

L'extrait montre par ailleurs JFC visant un objectif précis, au-delà de la pluralité des démarches possibles. Dans cette situation de projet, JFC se met dans la position des élèves pour voir ce qu'ils pourraient faire, la ressource est alors une aide pour compléter l'analyse de la situation mathématique :

«Euh, bon, j'ai vu aussi dans la ressource, aussi, différents modes de construction, il y a des idées que j'avais pas eues, c'est intéressant. » (entretien postérieur.)

Dans cette position de P-projeteur, JFC utilise la ressource pour affiner ses compétences à mettre en place une situation de recherche dans la classe. Il organise la séance en fonction de ce qu'il est capable de prévoir pour ses élèves spécifiques. JFC anticipant les différentes phases, envisage de placer ses élèves dans une position de chercheur, leur propose un milieu matériel riche:

« je vais leur présenter le sujet en leur donnant un document, euh ça se passera en salle informatique, j'ai fait ce choix et j'ai pensé au sujet d'ailleurs en même temps, au choix du sujet, j'ai pensé à la salle, je pense qu'ils utiliseront l'outil informatique » (entretien préalable.)

La ressource fournit également une aide pour le prolongement de la situation :

«Par la suite, puisque on n'a que 2 heures hein finalement à consacrer à ça, par la suite, éventuellement, j'exploiterai la ressource pour faire un petit complément, euh éventuellement sous forme d'un problème, qui sera pas trop ouvert quoi, en recherche à la maison par exemple. » (entretien préalable.)

Dans les entretiens menés, on perçoit bien les allers-retours entre les conceptions de l'enseignement et le projet de mise en œuvre effective dans une classe particulière. Comme élément du milieu du professeur, la ressource réagit aux propositions de séance en l'instrumentant et en participant à la construction de son ensemble documentaire.

Dans la situation didactique, le professeur présente à ses élèves la situation en suivant un scénario proposé dans la ressource. Il rajoute, conformément à sa position particulière de $\mathrm{P}$-noosphérien une réflexion sur la métacognition (cf. Fig. 5). JFC dans la situation didactique place ainsi rapidement ses élèves dans une situation surdidactique en prévoyant a priori un retour réflexif sur leur « aventure cognitive ».

Pendant la phase de recherche en groupe, P-observateur mesure la distance entre ses intentions en termes d'avancée du temps didactique et le travail effectif des élèves mais maintient une volonté de partir des propositions des élèves ; tous les dialogues montrent le tiraillement entre la volonté de faire émerger plus rapidement une paramétrisation du problème et la volonté de laisser les élèves explorer les pistes qu'ils souhaitent explorer (voir les productions des élèves en annexes 2 et 3 ) : 


\begin{abstract}
« J'ai quand même été un peu surpris que ce soit aussi long à démarrer et j'ai été aussi surpris, [...] j'ai été un peu surpris qu'ils ne paramètrent pas plus rapidement le problème. Euh eh bon ça c'est une bonne prise de conscience je le saurai c'est vrai qu'on est déformé par ce que l'on a l'habitude de faire et et du coup j'imaginais pas qu'ils auraient, qu'ils aient besoin d'un coup de main comme ça pour paramétrer euh.» (entretien postérieur.)
\end{abstract}

On peut cependant penser que le professeur agrandit dans cette posture son répertoire de réponses dans une telle situation de recherche de problème, et complète son système documentaire même si la ressource ne prévenait pas de cette possible difficulté :

« j'ai pas le souvenir d'avoir été alerté en quelque sorte dans la ressource par cette difficulté-là » (entretien postérieur.)

Dans la mise en place de la situation, JFC se place résolument dans une situation potentielle de la ressource et orchestre une situation de recherche qui rejoint sur de nombreux aspects les attentes des auteurs.

\section{Analyse d'un incident didactique}

Comme l'analyse descendante le laisse prévoir, JFC comme observateur se trouve confronté à une décision didactique c'est-à-dire " une décision prise avec une intention d'apprentissage par l'élève d'une connaissance visée » (Trgalova \& Lima, 2006). Comme nous l'avons déjà indiqué, JFC est surpris des directions de recherche prises par les élèves, et notamment qu'ils ne pensent pas (ou ne veulent pas, ou ne s'aventurent pas) à paramétrer le problème pour déboucher sur une étude analytique qui leur aurait permis de mettre en ouvre leurs connaissances des études de fonctions. Comme nous l'avons précédemment signalé, après 57 minutes, JFC interrompt les recherches :

«JFC : bien hein, excusez-moi, je vais vous interrompre un petit instant [...] ce qui est important, c'est de trouver un mode de construction de ce fameux point, et ce qui vous manque à tous, donc je vous fais remarquer, je vous donne cette information, c'est de choisir les paramètres ou des données pour définir votre figure. »
On peut considérer cette intervention comme un incident de contrat :

"Incident de contrat : un événement brise le contrat didactique ou le modifie sensiblement. Cette modification perturbe la trajectoire de la dynamique et est fortement reliée à l'apparition de bifurcations didactiques où les élèves investissent une situation nildidactique ou une perte locale de dévolution pendant laquelle un décrochement important peut être constaté. Tout au contraire, un incident de contrat peut relancer le travail dans une situation adidactique, pour peu que la responsabilité de l'apprentissage soit correctement dévolue; le contrat joue alors son rôle de régulateur des actions des élèves. » (Aldon, 2011, p. 67.)

Dans ce cas, il y a bifurcation, au sens où une branche différente de la branche principale de la situation visée par le professeur est empruntée, mais uniquement si nous considérons que JFC visait, à cet instant, une institutionnalisation sur la paramétrisation ou l'optimisation utilisant la dérivée d'une fonction d'une variable réelle et qu'il souhaitait que cet objet indiqué dans la ressource comme " potentiellement mobilisable " le devienne effectivement pour ses élèves. Mais si nous conservons l'idée que la situation didactique proposée par les auteurs est ouverte au travail de plusieurs savoirs et que JFC la voyait comme telle initialement, ce que l'entretien tend à montrer (cf. citations de JFC p. 22), l'incident didactique n'est pas déclencheur d'une bifurcation de construction mais, localement, interroge sur l'adaptabilité de cette situation de la ressource.

\section{Conclusion des analyses}

Les analyses précédentes montrent l'élaboration et la mise en œuvre par l'enseignant d'un projet qui rejoint sur de très nombreux points les intentions des auteurs de la ressource. L'absence globale de bifurcation de construction permet de conclure que la ressource est ici cohérente. Il se trouve que JFC a choisi la situation de la ressource repérée comme particulièrement délicate à mettre en œuvre, et qu'il s'est retrouvé, malgré une certaine expertise de la gestion de classe, en difficulté pour faire vivre des branches didactiques empruntées par certains groupes. JFC fait ponctuellement un choix didactique qui, à l'analyse, semble non pertinent. Toutefois, la 
cohérence de l'usage de la ressource résiste globalement à cet incident, les interactions entre l'enseignant et la ressource ayant produit une situation qui absorbe ce genre d'événements.

\section{CONCLUSION ET PERSPECTIVES}

À partir de la notion de milieu et de sa structuration issue de la théorie des situations didactiques, nous avons défini un concept de cohérence d'une ressource dans une situation d'enseignement ; la genèse documentaire a permis de construire une analyse du suivi de l'intégration d'une ressource dans l'ensemble documentaire d'un enseignant et d'en évaluer les effets dans la mise en ouvre d'une situation de classe. La ressource étudiée, EXPRIME, emblématique d'une ressource construite avec des intentions d'enseignement et d'apprentissage affirmées, met en avant le rôle de la dimension expérimentale des mathématiques dans la construction des connaissances des élèves. L'ingénierie construite pour mettre à l'épreuve ce concept de cohérence de l'usage a permis d'analyser cette ressource sous l'angle d'une intégration et d'une diffusion des intentions des auteurs dans l'enseignement des mathématiques. Comme il est signalé dans la conclusion de la partie précédente, les résultats de cette analyse montrent la cohérence de l'usage de la ressource au regard de la situation proposée par le professeur mais mettent aussi en exergue la complexité de la genèse documentaire et de l'intégration comme " document $»$ dans le système documentaire de l'enseignant.

Cette étude a permis de s'assurer de la possibilité de mettre en œuvre le concept de cohérence de l'usage d'une ressource et permet d'envisager désormais son utilisation dans des champs plus vastes. Au-delà de l'ingénierie spécifique décrite, le concept de cohérence de l'usage devra être mis à l'épreuve dans d'autres contextes, avec la même ressource, et avec d'autres ressources. Le premier champ d'expérimentation devra porter sur des contextes de classe ordinaire, c'est-à-dire en dehors de la construction d'une ingénierie spécifique. C'est en effet dans ce contexte que nous pourrons, à travers l'étude de la cohérence de l'usage d'une ressource, envisager d'élargir le propos à l'étude de la diffusion des ressources et de leur impact sur l'enseignement des mathématiques. De la même façon, un élargissement d'une telle étude à d'autres ressources devraient permettre de préciser et d'affiner ce concept de cohérence. Et alors que Margolinas (2005) insiste sur le fait que :

"Les situations incluant des bifurcations multiples et, à l'intérieur de celles-ci, des branches marginales nildidactiques, se révèlent très fréquentes à partir du moment où l'on a le cadre théorique et technique qui permet de les identifier. »

Il pourra être intéressant de voir si le concept de cohérence de l'usage met lui aussi en évidence une telle divergence entre les intentions des auteurs des ressources et les mises en œuvre qui en découlent. Dans cette optique, il pourrait être intéressant de voir comment ce concept de cohérence pourrait être affiné, par exemple en développant, au-delà de la notion de bifurcation / non-bifurcation utilisée ici, des degrés de fidélité aux intentions des auteurs.

D'autres questions émergent également de cette étude ; la ressource étudiée, aussi bien que l'analyse qui en est faite, placent la situation comme centrale en prenant le point de vue de la théorie des situations didactiques et il est légitime de se demander dans quelle mesure le concept de cohérence de l'usage dépend du cadre d'analyse didactique. Par ailleurs se pose la question de savoir si les ressources construites avec des intentions faiblement explicitées sont susceptibles de subir l'épreuve de la cohérence, et plus généralement de savoir quel champ d'étude peut couvrir cette notion et dans quelle mesure une bifurcation de projet peut constituer une instrumentalisation de la ressource lui permettant, dans un contexte spécifique, de devenir un élément du système documentaire du professeur au service de ses intentions didactiques.

\section{NOTES}

1. Trésor de la Langue Françoise informatisé, voir la page [http://atilf.atilf.fr/].

2. Institut de Recherche sur l'Enseignement des Mathématiques.

3. Institut de Recherche de l'Éducation.

4. Dernière année du secondaire, élèves de 17-18 ans.

5. Pour des analyses développées de cette situation, nous renvoyons à Aldon et al. (2010).

6. Avant-dernière année du secondaire, élèves de 16-17 ans. 7. JFC fait référence ici à des extraits d'une bande dessinée (Tisseron, 1984), présents dans la ressource EXPRIME. 


\section{RÉFÉRENCES}

Aldon, G. (2011). Interactions didactiques dans la classe de mathématiques en environnement numérique : construction et mise à l'épreuve d'un cadre d'analyse exploitant la notion d'incident (Thèse de doctorat, université Lyon 1 , France).

Aldon, G. (2014). Didactic incidents: a way to improve the professional development of mathematics teachers. Dans A. Clark-Wilson, O. Robutti \& N. Sinclair (Eds.), The Mathematics Teacher in the Digital Era: An International Perspective on Technology Focused Professional Development. Springer : Dordrecht.

Aldon, G., Cahuet, P.-Y., Durand-Guerrier, V., Front, M., Krieger, D., Mizony, M., \& Tardy, C. (2010). Expérimenter des problèmes de recherche innovants en mathématiques à l'école. Cédérom INRP.

Artigue, M. (1988). Ingénierie didactique. Recherches en didactique des mathématiques, 9(3), 281-208.

Arsac, G., Germain, G., \& Mante, M. (1988). Problème ouvert et Situation-problème. IREM de Lyon, université Claude-Bernard Lyon 1.

Arsac, G., \& Mante, M. (2007). Les pratiques du problème ouvert. Scéren CRDP : Lyon.

Arsac, G., \& Mante, M. (2008). Articulations clés dans l'histoire du problème ouvert. Communication, 11 janvier 2008 IREM de Lyon. Repéré à : [http://math. univ-lyon1.fr/irem/IMG/pdf/arsac.pdf ] (consulté le 24/02/14).

Bloch, I. (2005). Quelques apports de la théorie des situations à la didactique des mathématiques dans l'enseignement secondaire et supérieur (Habilitation à diriger des recherches, université Paris 7 , France).

Bloch, I., \& Gibel, P. (2011). Un modèle d'analyse des raisonnements dans les situations didactiques. Étude des niveaux de preuves dans une situation d'enseignement de la notion de limite. Recherches en didactique des mathématiques, 31(2), 191-228.

Boning, K. (2007). Coherence in general education: a historical look. Journal of general education, 56(1), $1-16$.

Bourgeat, F., Calpe, A., Digeon, M., Esfahani, E., Leyraud, I. et al. (2010). Working on resources quality assessment in i2geo. I2GEO 2010 conference Hluboka nad Vltavou (Czech republic). July, 2-3, 2010. Repéré à : [http:// cermat.org/i2geo2010/downloads/files/I2GEO2010Bourgeat.pdf] (consulté le 24/02/14).

Brousseau, G. (1986). Théorisation des phénomènes d'enseignement des mathématiques (Thèse de doctorat, université de Bordeaux 1, France).

Brousseau, G. (1998). Théorie des situations didactiques. Grenoble : La pensée sauvage éditions.

Clark-Wilson, A. (2010). How does a multi-representational mathematical ICT tool mediate teachers' mathematical and pedagogical knowledge concerning variance and invariance? (PHD., Institute of Education, University of London).
Durand-Guerrier, V. (2006). La résolution de problèmes, d'un point de vue didactique et épistémologique. Dans L. Trouche, V. Durand-Guerrier, C. Margolinas \& A. Mercier (Eds.), Actes des journées mathématiques de l'INRP (p. 17-23). Lyon : INRP.

Front, M. (2012). Pavages semi-réguliers du plan : une exploration favorable aux élaborations mathématiques. Repères IREM, 89, 5-37.

Gueudet, G., \& Trouche, L. (2008). Vers de nouveaux systèmes documentaires des professeurs de mathématiques. Dans F. Conne \& I. Bloch (Dir.), Actes de la $14^{e}$ École d'été de didactique des mathématiques.

Gueudet, G., \& Trouche, L. (2009). Towards new documentation systems for mathematics teachers? Education Studies in Mathematics, 71, 199-218.

Gueudet, G., \& Trouche, L. (2010). Ressources vives. INRP et Presses universitaires de Rennes.

Lakatos, I. (1984). Preuves et réfutations : essai sur la logique de la découverte mathématique. Hermann : Paris.

Mangiante-Orsola, C. (2012). Une étude de la cohérence en germe dans les pratiques de professeurs des écoles en formation initiale puis débutants. Recherches en Didactique des Mathématiques, 32(3), 289-331.

Margolinas, C. (2002). Situations, milieux, connaissances: analyse de l'activité du professeur. Dans J.-L. Dorier, M. Artaud, M. Artigue, R. Berthelot \& R. Floris (Éds.), Actes de la 11e École d'été de didactique des mathématiques (p. 141-156). Grenoble: La Pensée Sauvage éditions.

Margolinas, C. (2004). Points de vue de l'élève et du professeur. Essai de développement de la théorie des situations didactiques (Habilitation à diriger des recherches, université de Provence-Aix-Marseille 1, France).

Margolinas, C. (2005). Les bifurcations didactiques : un phénomène révélé par l'analyse de la structuration du milieu. Dans A. Mercier \& C. Margolinas (Éds). Balises en didactique des mathématiques (p. 1-12). Cédérom. La Pensée Sauvage.

Newmann, F.-M., Smith, B., Allensworth, E., \& Bryk, A.-S. (2001). Instructional program coherence : what it is and why it should guide school improvement policy. Educational Evaluation and Policy Analysis, 23(4), 297-321.

Owen, C. (2007). Analysing the activity of work in emergency incident management. @ctivités, 4(1), 217-225.

Pédauque, R. T. (2006). Le document à la lumière du numérique. Caen : C \& F éditions.

Perrin, D. (2007). L'expérimentation en mathématiques. Petit $x, 73,6-34$.

Polya, G. (1945). How to solve it. Princeton, N.J : Priceton University Press.

Rabardel, P. (1999). Éléments pour une approche instrumentale en didactique des mathématiques. Dans M. Bailleul (Ed.), Actes de la Xe École d'été de didactique des mathématiques (p. 202-213). Houlgate: IUFM de Caen. 
Roditi, E. (2001). L'enseignement de la multiplication des décimaux en sixième, étude de pratiques ordinaires (Thèse de doctorat, université Paris 7, France).

Rogalski, J. (2000). Approche de psychologie ergonomique de l'activité de l'enseignant. Dans Actes du XXVI $I^{\mathrm{e}}$ colloque COPIRELEM (p. 45-66).

Schmidt, W.-H, Hsing, C., \& McKnight, C.-C. (2005). Curriculum coherence: An examination of US mathematics and science content standards from an international perspective. Journal of Curriculum Studies, 37(5), 525-559.

Schoenfeld, A.-H. (1985). Mathematical Problem Solving. New York : Academic Press.

Tisseron, C. (1984). La pratique du problème ouvert. Adaptation Mizony (2005) : Repéré à [http://irem-fpb. univ-lyonl.fr/feuillesprobleme/feuille3/bd/html/pbouvert.html] (consulté le 24/02/14).

Trgalova, J., \& Lima, I. (2006). Didactic decisions: the case of reflexion. Dans J. Novotna, H. Moraova, H. Kratka \&
N. Stehlikova (Eds.), Proceedings 30th Conference of the International Group for the Psychology of Mathematics Education (p. 343). Prague, 16-21 July.

Trgalova, J., \& Leyraud, I. (2010). Ressources, critères de qualité pour la conception et les usages. Dans G. Gueude, G. Aldon, J. Douaire, J. Trgalova (Dir.), Apprendre, Enseigner, se former en mathématiques : quels effets des ressources? Actes des journées mathématiques de l'INRP (p. 139-144). Éditions électroniques, ENS de Lyon, [http://ife.ens-lyon.fr/editions/editions-electroniques/apprendre-enseigner-se-former-en-mathematiques] (consulté le 24/02/14).

Tricot, A., Plégat-Soutjis, F., Camps, J.-F., Lutz, A. A. G., \& Morcillo, A. (2003). Utilité, utilisabilité, acceptabilité : interpréter les relations entre trois dimensions de l'évaluation des EIAH. Dans C. Desmoulins, P. Marquet $\&$ D. Bouhineau (dir.), Environnements informatiques pour l'apprentissage humain (p. 391-402). Paris : ATIEF - INRP. 


\section{ANNEXE 1 : LA RESSOURCE EXPRIME}

La ressource EXPRIME est une ressource numérique qui permet une lecture non linéaire et des entrées variées suivant les intentions du lecteur. Une page d'accueil propose un premier menu par lequel il est possible :

- de parcourir des textes théoriques concernant la dimension expérimentale en mathématique (Kuntz 2007 ; Durand-Guerrier 2006) et des présentations faites dans des colloques et conférences;

- de comprendre l'esprit de la ressource en parcourant une présentation générale et son historique ;

- d'accéder aux situations qui sont présentées suivant une structure commune ;

- de participer au forum des utilisateurs.

Les situations mathématiques de la ressource sont actuellement au nombre de sept :

1. Les fractions égyptiennes. Décomposer l'unité en somme de fractions de numérateurs un.

2. Les nombres trapézoïdaux. Étude de sommes d'entiers consécutifs.

3. La rivière. Étude du plus court chemin d'un point à un autre passant par une courbe donnée.

4. Une intersection inaccessible. Sachant que le point d'intersection de deux droites est inaccessible, trouver une droite passant par ce point.

5. Le nombre de zéros de $n$ !. Étude des chiffres de $n$ ! dans un système de numération donné.

6. Le plus grand produit. Étude du produit de nombres entiers à somme fixée.

7. Les urnes de Polya. Étude de la dynamique de la composition d'une urne dans une expérience répétée. Elles sont construites et présentées suivant une structure commune :

Situation mathématique : il s'agit d'une analyse a priori du problème mathématique et de ses solutions, sans tenir compte d'un niveau particulier de classe. Objets mathématiques potentiellement travaillés : cette entrée permet de savoir quels sont les objets mathématiques (concepts, notions, outils, démarches) que les enseignants peuvent s'attendre à voir émerger des travaux des élèves. Les auteurs s'appuient sur des observations de classe pour faire ressortir les invariants qui permettent de prévoir, à un niveau de connaissance donné, les obstacles rencontrés par les élèves, mais aussi les savoirs et connaissances sur lesquels les enseignants pourront construire l'institutionnalisation.

Situations d'apprentissage : des scénarios adaptés à des niveaux de classe, un ou plusieurs énoncés, la description des variables didactiques de la situation proposée ainsi que des comptes-rendus d'observations de classes permettent de mettre en évidence les réactions des élèves mis dans une démarche de recherche de problème.

Références: les situations proposées ne cherchent pas l'originalité et nous présentons dans cette partie d'autres approches de la situation, sur le web ou dans la littérature.

Synthèse : un condensé de quelques pages permettant de prendre connaissance de la situation et des objectifs d'apprentissage.

Situations connexes : il s'agit de mettre en évidence les prolongements possibles de la situation mathématique pour permettre la construction de nouvelles situations didactiques dans les classes.

Ainsi, à partir d'une situation mathématique s'organisent des situations de classes (énoncés, scénarios et compte rendus d'expérimentation) complétées par des références et une fiche de synthèse de la situation. Cependant, le fait que cette ressource soit numérique permet une lecture non linéaire et des entrées variées suivant les intentions du lecteur.

Figure 6.

Structure de la ressource EXPRIME

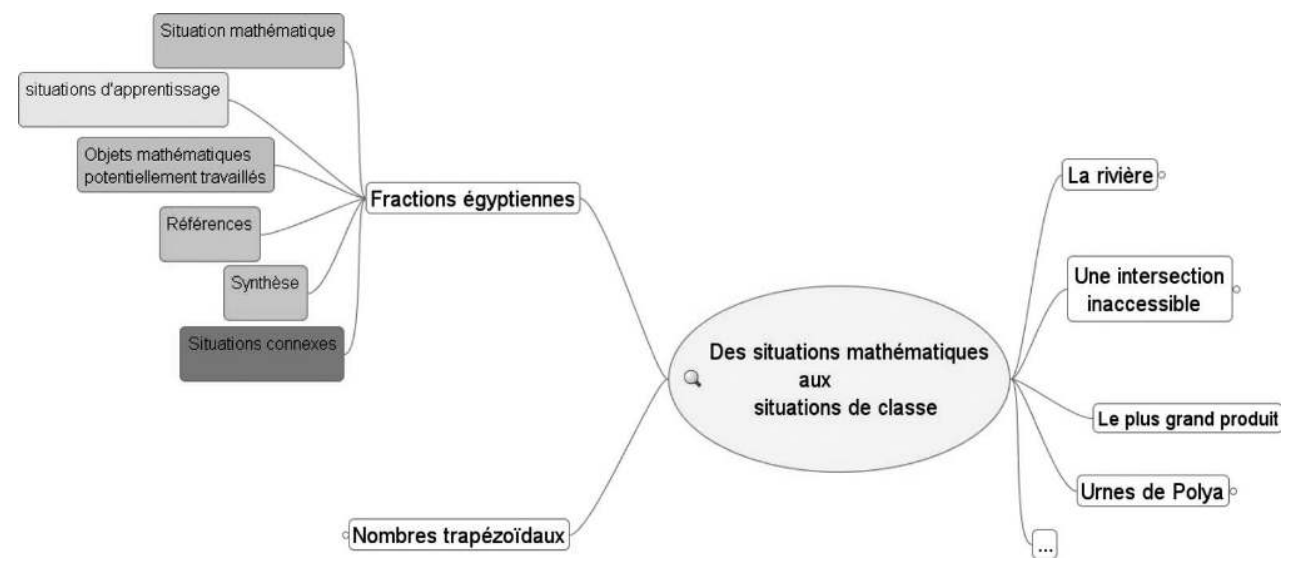




\section{ANNEXE 2 : TRANSPARENT DU GROUPE 1}

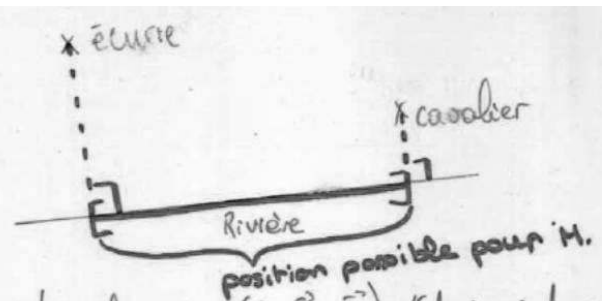

On considedr le repére $(0 ; \vec{i}, \vec{j})$ défini ci-dessous:

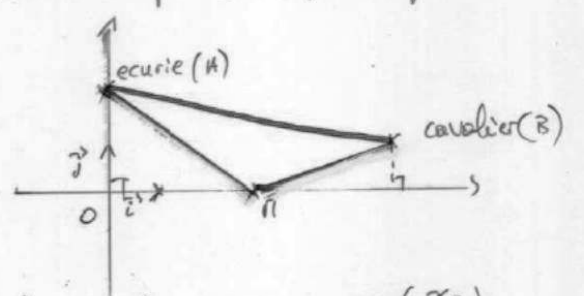

$$
A\left(\begin{array}{l}
0 \\
y_{n}
\end{array}\right) \quad B\left(\begin{array}{l}
x_{B} \\
k y_{a}
\end{array}\right), k \in R \quad \pi\left(\begin{array}{l}
x_{n} \\
0
\end{array}\right)
$$

on veut $\pi, A \Pi+B \pi$ plus petit possible.

$A n^{2}=x_{n}^{2}+y_{a}^{2}$

$\pi \theta^{2}=\left(x_{\beta}-x_{n}\right)^{2}+\left(b y_{a}\right)^{2}$

Dore $A n+n B=\sqrt{x_{n^{2}}{ }^{2}+y_{Q^{2}}}+\sqrt{\left(x_{B}-x_{n}\right)^{2}+\left(k_{1 / a}\right.}$
ANNEXE 3 : TRANSPARENT DU GROUPE 2

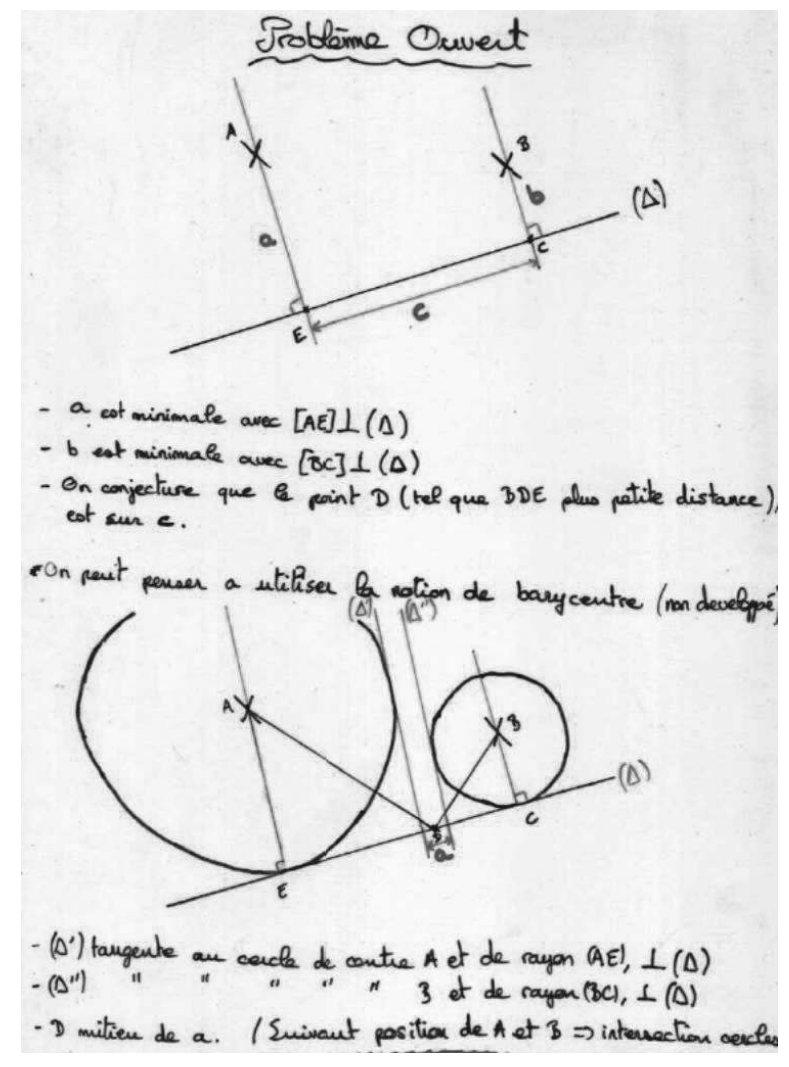

